\title{
Recurrent spontaneous abortion and polycystic ovarian disease: comparison of two regimens to induce ovulation
}

\author{
Pam Johnson, J Malcolm Pearce
}

\begin{abstract}
Objective-To determine whether pituitary suppression before induction of ovulation reduces the rate of spontaneous abortion in women with polycystic ovarian disease and primary recurrent spontaneous abortions.
\end{abstract}

Design-Closed, randomised, sequential trial. Pairs of women were allocated to each treatment by the toss of a coin.

Setting-Supraregional clinic for women who had had recurrent spontaneous abortions.

Subjects-Forty two women with polycystic ovarian disease and primary recurrent spontaneous abortions.

Interventions - Ovulation was induced by clomiphene or pituitary suppression with buserelin followed by pure follicle stimulating hormone.

Main outcome measures-Preference for a particular treatment was noted. A preference occurred when one woman in a pair had a successful pregnancy (defined as one of over 12 weeks' gestation) and one had a spontaneous abortion; the preference was for the treatment resulting in the successful pregnancy.

Results-Spontaneous abortions occurred in 11 of 20 women given clomiphene compared with two of 20 who had pituitary suppression. Eleven preferences were found for buserelin and two for clomiphene. In seven pairs both women had successful pregnancies. One pair was discarded because one of the women did not become pregnant. The ratio of luteinising hormone concentration to follicular diameter was found to be a possible diagnostic indicator of spontaneous abortion.

Conclusion-Pituitary suppression before induction of ovulation significantly reduces the risk of spontaneous abortion in women with polycystic ovarian disease and primary recurrent spontaneous abortions.

\section{Introduction}

Patients with polycystic ovarian disease who require treatment with clomiphene to induce ovulation are at increased risk of early spontaneous abortion, which may occur in $40-50 \%$ of pregnancies. ${ }^{12}$ The high basal concentrations of luteinising hormone that occur in polycystic ovarian disease are thought to affect chances of conception and successful pregnancy adversely. ${ }^{3} \mathrm{We}$ compared the rate of spontaneous abortion after induction of ovulation with either clomiphene or follicle stimulating hormone after pituitary suppression in women with polycystic ovarian disease who had had primary recurrent spontaneous abortions.

and Gynaecology, St

George's Hospital, London SW17

Pam Johnson, MRCOG, registrar

J Malcolm Pearce, FRCS, senior lecturer

Correspondence to: $\mathrm{Mr}$ Pearce.
Women with polycystic ovarian disease, who had a history of recurrent spontaneous abortion, had conceived during previous treatment with clomiphene, and were not pregnant were eligible for the study. Polycystic ovarian disease was diagnosed if the ratio of luteinising hormone to follicle stimulating hormone concentration was $>3$ and ultrasonography showed multiple cysts of $2-8 \mathrm{~mm}$ diameter either peripherally or scattered throughout an increased stroma. ${ }^{+}$The women were classified as having had primary recurrent spontaneous abortions if they had had three or more unexplained spontaneous abortions during the first trimester without an intervening term pregnancy that reached term. Each miscarriage had to have been documented by at least one of the following: positive result of a pregnancy test, ultrasonography, or histological examination of the products of conception. The women were recruited from a clinic for women who had early spontaneous abortions, which attracted women on a supraregional basis.

All the women studied had a normal karyotype and yielded negative results to tests for thyroid dysfunction, lupus anticoagulant (both between pregnancies and in the pregnancy being studied), and antibodies to chlamydia, brucella, toxoplasma, and cytomegalovirus. In addition, cultures from cervical swabs did not disclose herpes simplex or mycoplasma in any woman and monoclonal antibody tests for chlamydia gave negative results. None of the women had anaemia or abnormal blood group antibodies. Their husbands had normal karyotypes, and none had polyspermia.

The study was designed as a closed, randomised, sequential trial. As the estimated background miscarriage rate was $40 \%$ we considered that a reduction to $20 \%$ in the group having pituitary suppression would be clinically important. The maximum number of preferences needed to show this at a $5 \%$ significance level $(\alpha=0.05)$ was calculated to be 27.5 Pairs of patients were allocated to one or other treatment groups by the toss of a coin. A preference was considered to have occurred when both members of the pair became pregnant but one member miscarried. A preference was then recorded for the treatment that resulted in the successful pregnancy. The study was blind only so far as the staff who performed pregnancy tests and ultrasonography were unaware of the patients' treatment.

Pairs of women were allocated to have ovulation induced with clomiphene citrate or pituitary suppression with buserelin followed by pure follicle stimulating hormone (by tossing a coin). The women allocated to the clomiphene group received 50-150 mg clomiphene daily on days 2-6 of their natural cycle, the dose depending on that which had previously resulted in pregnancy and which produced ultrasonic evidence of ovulation. The serum luteinising hormone concentration was measured once in each patient, at the time of maximum growth of the follicle, by radioimmunoassay (using the Chelsea reagents) as part of a routine clinical service. The variation between assays for the luteinising hormone concentrations reported in the study (5$35 \mathrm{IU} / \mathrm{l})$ was less than $6 \%$.

The group having pituitary suppression was given $200 \mu \mathrm{g}$ buserelin four hourly by intranasal spray. The treatment was continued for a minimum of three weeks until serum oestradiol concentration was less than $100 \mathrm{pmol} / \mathrm{l}$. They were then given pure follicle stimulating hormone, the dose depending on the response seen by ultrasonography on alternate days. When the 
leading follicle was $18-20 \mathrm{~mm}$ diameter the women were given 10000 units of human chorionic gonadotrophin and the buserelin was stopped. Ultrasonography was performed with a Combison 320 machine (Kretztechnik) with a $3.5 \mathrm{MHz}$ transabdominal sector scanner. The coefficient of variation between observers for measurements of follicular diameter was of the order of $9 \%$.

Pregnancies were confirmed by an assay of urinary human $\beta$-chorionic gonadotrophin performed at least four days after a missed period and dectection of an intrauterine gestation sac on ultrasonography. Pregnancies were considered to be successful if they exceeded 12 weeks' gestation.

\section{Results}

Twenty one pairs of women were recruited, but one pair was discarded because the woman receiving buserelin did not become pregnant after 12 cycles. There were no demographic differences between the two groups of 20 women (table I). We did not need to recruit further women as the upper limit for significance was crossed after 13 preferences.

Figure 1 shows the sequential design of the study.

TABLE I-Demographic details of women studied according to treat ment received

\begin{tabular}{lcc}
\hline & $\begin{array}{c}\text { Buserelin } \\
(\mathbf{n}=20)\end{array}$ & $\begin{array}{c}\text { Clomiphene } \\
(\mathbf{n}=20)\end{array}$ \\
\hline $\begin{array}{l}\text { Mean (SD) age (years) } \\
\text { Mean No of previous spontaneous }\end{array}$ & $31 \cdot 0(5 \cdot 8)$ & $30 \cdot 8(6 \cdot 1)$ \\
$\quad$ abortions (range) & $3 \cdot 3(3-6)$ & $3 \cdot 3(3-5)$ \\
Racial group: & 2 & 2 \\
$\quad$ Asian & 3 & 3 \\
$\quad$ Black & 15 & 14 \\
\hline
\end{tabular}

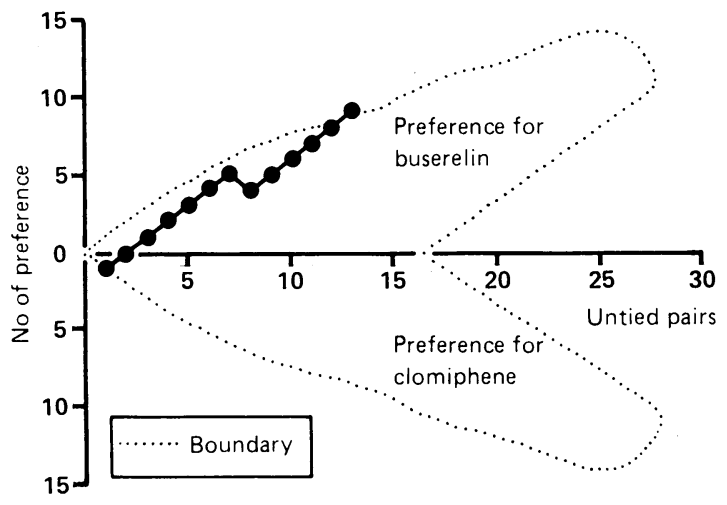

FIG $1-C l o s e d$ sequential design of trial. A preference was considered to have occurred when one woman of a pair randomised to receive buserelin or clomiphene had a spontaneous abortion. The y axis indicates preference for either buserelin and follicle stimulating hormone or clomiphene. The pair of women were plotted in an upward direction if the successful pregnancy was achieved with buserelin and in a downward direction if it was achieved with clomiphene. Pairs in which a preference did not occur were not plotted. The trial was stopped when the boundary, determined according to the method given by Armitage, was crossed

Each pair of patients in which a preference occurred was entered on the diagram. In seven pairs both women had a successful pregnancy, so that a preference did not occur. Eleven of the women given clomiphene had a spontaneous abortion compared with two given buserelin; this was significant at the $1 \%$ level $(95 \%$ confidence interval for difference in proportions having spontaneous abortions, $19 \%$ to $71 \%$ ).

The number of menstrual cycles required to achieve pregnancy was similar in both groups (mean $2 \cdot 4$ ). One woman in each of the treatment groups required six cycles. Fifteen cycles were abandoned in the group who received buserelin, five because three or more follicles developed and 10 because no dominant follicle developed. No cycles were abandoned in the group given clomiphene.

We defined a successful pregnancy as one that exceeded 12 weeks' gestation. No mid-trimester losses occurred, however, and all pregnancies that exceeded 12 weeks' gestation resulted in live births. While we were waiting for the 40th woman recruited to the study to achieve 12 weeks' gestation three more pairs of women were entered into the study, but none of these women had a spontaneous abortion. This gives an overall miscarriage rate of $48 \%(11 / 23)$ for the group given clomiphene compared with $9 \%(2 / 23)$ for the group given buserelin.

An additional finding in the group given clomiphene was the possible value of the ratio of luteinising hormone to follicular diameter as an indicator of the likelihood of spontaneous abortion. Figure 2 shows a scatter plot of this ratio according to outcome. No pregnancy progressed into the second trimester if the ratio was greater than $0 \cdot 8$.

\section{Discussion}

Clomiphene occupies oestrogen receptors in the hypothalamus and increases the frequency of the pulses releasing both follicle stimulating hormone and luteinising hormone, ${ }^{6-}$ thereby increasing their serum concentrations. It has a long half life, being detectable for up to 28 days after a single $50 \mathrm{mg}$ dose. ${ }^{8}$ The rate of spontaneous abortion in pregnancies induced by clomiphene in women with polycystic ovarian disease is at least $40 \%$.' $^{\prime}$ Possible reasons for this high rate are the antioestrogen action of clomiphene, which, because of its long half life, may cause defective production of progesterone in the luteal phase, $(\mathrm{G}$ Lavy et al, meeting of the American Fertility Society, New York, 1986) and the high basal concentrations of luteinising hormone found in polycystic ovarian disease, which inhibit oocyte maturation inhibitor. ${ }^{9}$ Oocyte maturation inhibitor is produced by granulosa cells and maintains the primordial follicle in the diplotene phase of meiosis. It is inhibited by increased cyclic AMP concentration, which depends on luteinising hormone concentrations. Premature reactivation of meiosis caused by high basal concentrations of luteinising hormone may lead to karyotypic abnormalities and death of the embryo and fetus. We were unable to confirm this theory in our study because our patients were referred from all over the South West Thames region. The high rate of pregnancy loss in women with high basal concentrations of luteinising hormone is well recognised. ${ }^{10}$

We could not blind the study because clomiphene was taken orally for five days whereas buserelin was taken as an intranasal spray for a minimum of three weeks and accompanied by injections of follicle stimulating hormone. Patients and doctors were, therefore, aware of the treatments. The ultrasonographers who confirmed the pregnancies and spontaneous abortions, however, were unaware of which group the patients belonged to. After pregnancy had been confirmed all patients were managed by clinicians who were not participating in the trial. We therefore do not believe that unavoidable knowledge of treatment affected the number of spontaneous abortions.

Our study clearly shows that pituitary suppression followed by induction of ovulation with follicle stimulating hormone and human chorionic gonadotrophin increases the chance of a successful pregnancy in women who have polycystic ovarian disease and recurrent spontaneous abortions. The regimen, however, is very labour intensive and entails a large commitment by both the patient and the doctor. It is luteinising hormone concentration to follicular diameter 
also expensive (clomiphene costs $£ 3.50$ - $£ 4.75$ each cycle whereas buserelin and follicle stimulating hormone cost $£ 451$ - $£ 2307$ each cycle), which makes it difficult to recommend this as the primary method of inducing ovulation in women with polycystic ovarian disease. If, however, the value of the ratio of luteinising hormone concentration to follicular diameter in predicting spontaneous abortions is confirmed this could be used to monitor women given clomiphene to induce ovulation. If high ratios were found the women could be advised to use barrier methods of contraception, and if a high ratio occurred in successive cycles pituitary suppression could be started.

Maggregor $\mathrm{AH}$, Johnson JE, Bunde $\mathrm{CA}$. Further clinical experience with clomiphene ciurate Ferul Suril 1968:19:616-20.
2 Yen SSC. The polycystic ovary syndrome. (lin Endocrnol 1980;12:177-208. Homburg R, Armar NA, Eshel A, Adams J, Jacobs HS. Influence of serum luteinising hormone concentrations on ovulation, conception, and early pregnancy loss in polycystic ovary sundrome. Br Med f 1988:297:102t-6.

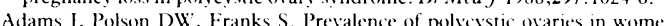

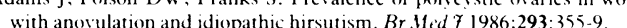
whe

Kerin JF, Lui JH, Phillipou G, Yen SSC. Evidence for a hypothalamic site of action of clomiphene citrate in women. 7 Clin Endocrinol Metah 1985;61:265.

7 Judd SJ, Alderman J, Bowden J, Michailov L. Evidence of involvement of opiate neurons in mediating the effect of clomiphene citrate on gonadotrophin-releasing hormone neurons. Fertil Steril 1987;47:574-8.

8 Mikkelson TJ. Single dose pharmacokinetics of clomiphene citrate in normal volunteers. Fertil Steril 1986:46:392-6

9 Tsafriri A. Pomerantz SH. Ooçte maturation inhibitor. Clm Endocrnol 1986:15:157-70.

10 Stanger JD, Yovitch JL. Reduced in vitro fertilisation of human oocytes from patients with raised hasal luteinising hormone levels during the follicular phase. Br 7 Obstet (junaecol 1985;92:385-90.

Accepted 9 November 1989

\title{
Serological diagnosis of infection with human herpesvirus type 6
}

\author{
William L Irving, Anthony L Cunningham
}

\begin{abstract}
Objective-To identify clinical consequences of acute human herpesvirus type 6 infection by hypothesising that the virus will induce similar clinical syndromes to cytomegalovirus.

Design-Examination of consecutive serum samples from patients with illnesses compatible with acute cytomegalovirus infection or exanthem subitum by indirect immunofluorescence for the presence of antibodies to human herpesvirus type 6. An IgG absorption step was included to avoid false positive and negative results for IgM. The criterion standard for diagnosis of human herpesvirus type 6 infection was the presence of IgM human herpesvirus type 6 antibody (titre $>20$ ) and a rising titre of IgG human herpesvirus type 6 antibody without serological evidence of alternative infection.
\end{abstract}

Setting-Routine viral diagnostic and reference laboratory in the largest teaching hospital in Sydney.

Patients-341 Consecutive serum samples were analysed from patients with hepatitis (147 samples); infectious mononucleosis-like illness (106); screens for toxoplasma, other viruses, rubella, cytomegalovirus, and herpesvirus (38); fever in an immunocompromised patient (eight); unusual neurological (nine) or haematological syndromes (14); splenomegaly (six); and rash in a child (13).

Results - Three cases of acute human herpesvirus type 6 infection were identified: in one patient aged 65 with a previous diagnosis of acute non-A non-B hepatitis, one aged 25 with a glandular fever-like illness, and one aged 6 with a glandular fever-like illness. All three illnesses resolved completely. 15 Further serum samples were positive for human herpesvirus type 6 antibody but were also diagnostic for acute infection with other viruses (cytomegalovirus (nine), Epstein-Barr virus (three), and HIV (one)) or had a titre of IgM human herpesvirus type 6 antibody $<\mathbf{2 0}$ (two).

Conclusions-Acute human herpesvirus type 6 infection in immunocompetent patients may result in a mononucleosis-like illness or an acute but self limiting hepatitis.

\section{Introduction}

Human herpesvirus type 6 is a recently identified human lymphotropic virus which shows tropisms for diverse cells in vitro. ${ }^{1-3}$ Serological surveys suggest that most adults have been infected by the virus. ${ }^{+}$Primary infection with the virus in infancy may result in exanthem subitum (roseola infantum) ; $^{\text {; }}$ possible clinical consequences of primary infection in older children and adults have not been fully defined.

Among the human herpesviruses, the type 6 virus most resembles cytomegalovirus both morphologically and in genomic homology. ${ }^{67}$ By hypothesising that the virus is likely to behave biologically similarly to cytomegalovirus we selected serum samples arriving in this diagnostic laboratory to screen for evidence of acute infection with human herpesvirus type 6 . The initial sceening strategy was designed to identify not only serum samples containing IgM human herpesvirus type 6 antibody but also those with high titre specific IgG antibody, as such samples might give rise to false negative results for IgM antibody. Serum samples positive on screen testing were then retested for the presence of IgM human herpesvirus type 6 antibody after an IgG absorption step. We describe our initial experience with this strategy, which resulted in the identification of three patients whose illness coincided with serological evidence of acute infection with human herpesvirus type 6 .

\section{Samples and methods}

Serum samples from patients with a history compatible with either an acute cytomegalovirus infection or exanthem subitum were screened. The clinical details were categorised as: (a) hepatitis ( 147 samples); (b) infectious mononucleosis-like illness (106); (c) screen for toxoplasma, other viruses, rubella, cytomegalovirus, and herpesvirus ( 25 cord and 13 maternal samples); (d) fever in an immunocompromised patient (eight); (e) unusual neurological syndromes (nine); $(f)$ unusual haematological syndromes $(14)$; $(g)$ splenomegaly (six); and $(h)$ unexplained rash in a child (13). The samples were selected after the requested diagnostic tests had been performed, thus eliminating those in which an alternative virological cause had already been diagnosed.

The samples were screened by indirect immunofluorescence for the presence of IgM human herpesvirus type 6 antibody at a dilution of one in 20 and for high titre IgG human herpesvirus type 6 antibody at one in 100 dilution with acetone fixed J Jhan cells infected with human herpesvirus type 6 as substrate. Fluorescein conjugated sheep antihuman immunoglobulin (Wellcome) was used as the second antibody for IgG and $F\left(a^{\prime}\right)_{2}$ fragment goat antihuman IgM 